\title{
Genetic testing for hereditary breast cancer in Asia-moving forward
}

\author{
Ava Kwong ${ }^{1,2,3}$ \\ ${ }^{1}$ Department of Surgery, The University of Hong Kong, Queen Mary Hospital, Hong Kong; ${ }^{2}$ Department of Surgery, Hong Kong Sanatorium \& \\ Hospital, Hong Kong; ${ }^{3}$ Hong Kong Hereditary Breast Cancer Family Registry, Hong Kong \\ Correspondence to: Dr. Ava Kwong. Chief of Breast Surgery Division, The University of Hong Kong, Chairman, The Hong Kong Hereditary Breast \\ Cancer Family Registry, Room K1401, Queen Mary Hospital, Pokfulam Road, Hong Kong. Email: akwong@asiabreastregistry.com; avakwong@hku.hk.
}

\begin{abstract}
Genetic screening for hereditary breast and ovarian cancer (HBOC) has gained much attention for the past decades. With the development of advanced sequencing technology, other novel breast cancer associated susceptibility genes, other than BRCA genes, have been identified recently. The prevalence of $B R C A$ mutation is known to be different in the West and in the East, therefore it is important to understand the mutation spectrum locally and in Asia to improve early diagnosis and clinical management of hereditary breast cancer in the region. In this editorial, we sought to highlight the need of genetic screening in HBOC, and also highlight specific issues within Asia which may need to be addressed to help popularize its appropriate use in this region.
\end{abstract}

Keywords: Asia; breast cancer; genetic testing; ovarian cancer

Submitted May 28, 2016. Accepted for publication May 30, 2016.

doi: $10.21037 /$ cco.2016.05.11

View this article at: http://dx.doi.org/10.21037/cco.2016.05.11

\section{Introduction}

Breast cancer is the most common cancer worldwide and the second most common leading cause of cancer related death among women. Although incidence is generally lower in Asia, it is still the most prevalent female cancer in this part of the world. It is estimated that at least $60 \%$ of the world's 7 billion people are Asians and in particular the fast growing trend of breast cancer incidence in Asia especially China and India where the population is large can impose significant health burden to the society (1). There is an estimated 1.67 million new cases of breast cancer diagnosed in 2012 worldwide and 39\% of these are found in Asia (Globocan 2012, http://globocan.iarc.fr/Pages/fact_ sheets_cancer.aspx). There is increasing knowledge that a proportion of breast cancer are due to the inheritance of mutations in two susceptibility high penetrant genes, $B R C A 1$ and BRCA2 (2). Women who carry such genetic alterations in $B R C A 1$ and $B R C A 2$ genes has four to five fold increased lifetime risk of developing breast cancer by the age of 70 years compared to general population (45-85\% vs. $12.5 \%$ ) (3). Although most studies on BRCA mutations have been performed in Caucasian or Ashkenazi Jewish cohorts, there are increasing literatures published over the past few years where individual Asian countries have similar frequencies of $B R C A 1 / 2$ mutations in clinically high risk cohorts which have been tested (4).

Moreover, for decades, the use of PCR based Sanger Sequencing and additional methodology such as multiplex ligation-dependent probe amplification (MLPA) has been the gold standard technique in identifying germline and hotspots mutations (5). With the advent of advanced technology, next-generation sequencing (NGS) based on clonal amplifications coupled with massively parallel sequencing has become more commonly used. This allows the investigation of multiple genes at a lower cost and a faster turnover time (5-7) and facilitates the testing of additional gene mutations both in the germline and somatic setting, hence a huge influx of multigene panels testing has become available both in the commercial setting and academic setting (7-10).

With the increase awareness and knowledge of hereditary breast and ovarian cancer (HBOC), i.e., reduction of cost, turnover time and availability of genetic testing, it is inevitable that genetic testing will have an increase role in Asia. However, the implementation of any health care 
Table 1 Hereditary breast and/or ovarian cancer syndrome testing criteria (adapted from NCCN Guidelines Version 2.2016)

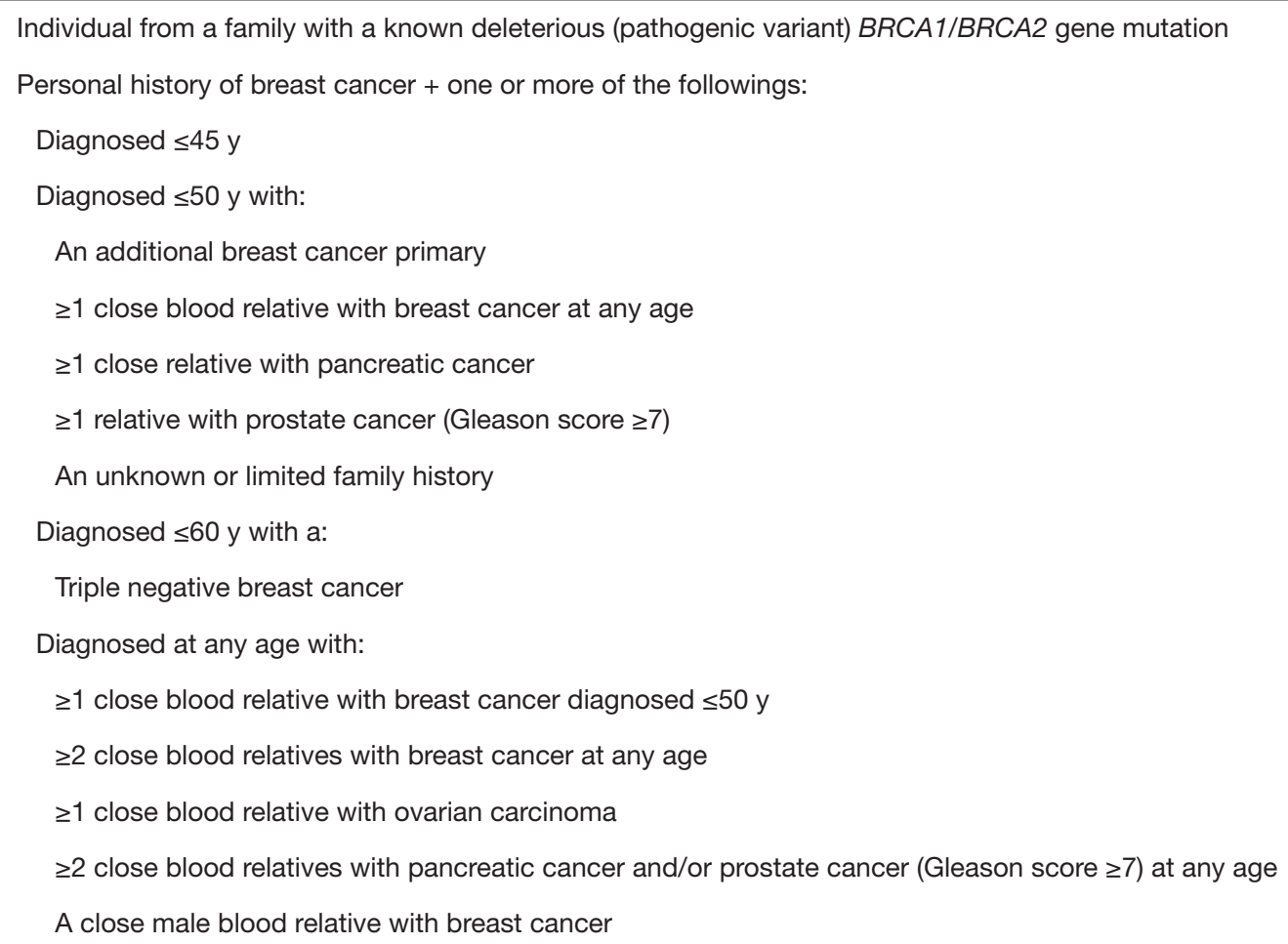

For an individual of ethnicity associated with higher mutation frequency (e.g., Ashkenazi Jewish) no additional family history may be required

Personal history of ovarian carcinoma

Personal history of male breast cancer

Personal history of pancreatic cancer or prostate cancer (Gleason score $\geq 7$ ) at any age with $\geq 1$ close blood relative with ovarian carcinoma at any age or breast cancer $\leq 50$ y or two relatives with breast, pancreatic or prostate cancer (Gleason score $\geq 7$ ) at any age

Personal history of pancreatic cancer and Ashkenazi Jewish ancestry

Family history only

First- or second-degree blood relative meeting any of the above criteria

Third-degree blood relative with breast cancer and/or ovarian carcinoma with $\geq 2$ close blood relatives with breast cancer (at least one with breast cancer $\leq 50 \mathrm{y}$ ) and/or ovarian carcinoma

NCCN, National Comprehensive Cancer Network.

options less known ethnic cohort may require adaptations and adjustments to established guidelines set for use in Western countries.

The selections for genetic testing of individuals are fairly well defined in Western countries. For mutations such as BRCA1 and BRCA2 mutations or even other high penetrant genes, selection of individuals for genetic testing are mainly based on age of onset of breast or ovarian cancer, the extend of family history of related cancers, and also subtype of cancers, for example triple negative breast cancer for $B R C A$ mutations.

The most well established guidelines would be that of National Comprehensive Cancer Network (NCCN) Version 2.2016 (Table 1). Apart from the clinical indications based on cancer history, a number of genetic risk prediction models have been established, [such as BOADICEA, BRCAPRO, Myriad, Couch (also known as PENN), Shattuck-Eidens and Manchester (11-16)] that can assess 
the pre-test probability of identifying a $B R C A 1$ or $B R C A 2$ mutation and enable efficient targeting of genetic testing. However these models are mainly designed based on nonAsian cohorts and a number of Asian countries have found the variability of the accuracy in using these models on Asian cohorts leading to the development of Asian based models such as KOHCal which is at present being validated on other Asia cohorts and aims to increase the accuracy of selection of appropriate probands for genetic testing (17). Most Asian countries however would base their selection of probands by clinical criteria (4).

The management of mutation carriers particularly for $B R C A$ mutations is also very well established and for the past two decades emphasis have been on both primary and second prevention, namely screening to achieve early diagnosis or aiming for risk reduction using surgery or medication. For the breasts, mutation carriers for $B R C A$ mutations are usually offered alternate screening options including the choice of intensive breast surveillance (18) (MRI breasts in addition to standard breast imaging such as mammography and ultrasonography), and preventative interventions including the consideration of mastectomy instead of breast conservation surgery due to the increased risk of contralateral breast cancer and the likelihood of increase second primary cancers in the ipsilateral breast in the setting where the individual have already had breast cancer. For risk reduction, options of prophylactic mastectomy and salpingo-oophorectomy, or the prescription of chemoprevention drugs are the main choices. The indications of $B R C A$ mutation testing have become more popularized recently as new data have supported the options of selection of therapeutic management of breast and ovarian cancers based on BRCA mutation status such as the choice of chemotherapy as primary treatments such as the use of carboplatin as a neoadjuvant agent and the evolvement of the use of targeted therapies, such as PARP inhibitors.

However the uptake rate and choice of management in Asia is much dependent on the availability of screening options and MRI are less likely performed in most Asian countries due to lack of resource or availability. Prophylactic surgeries are offered but uptake rates of RRM are much less compared to United States (1).

Genetic counselling plays an important role in selection of individuals suitable for genetic testing, collecting an accurate family pedigree and to explain the process, advantages and disadvantages of genetic testing and its implication not only to the individual but also the family members. Traditional counselling takes a long period of explanation on an individual face to face basis. A genetic counselling requires a 1-2 years training in Western Universities in general but in Asia there is a lack of training for genetic counselling aside from larger countries such as Japan and Korea where there are national and regional training programmes for genetic counsellors $(19,20)$. For most other countries genetic counsellor are usually nurses, psychologist or other allied health workers with certification training in cancer genetics from long distance certification courses or trained by the genetic specialist in the clinical setting locally. With the increase indications for genetic testing, it is likely that like the West, the Asian model of counselling may also have to change base on the needs and local adaptation. The develop of the best implementation of counselling flow will need to be addressed be it the choice of traditional counselling, or newer models using telephone counselling, streamlining other specialties such as oncologist based initial counselling but yet avoiding the misinterpretation of the genetic result and compromising care, would be of utmost importance.

It has also been established that although the prevalence of mutations in genes such as BRCA genes are likely to be similar among different ethnicities, based on studies on $B R C A$ mutations in Asian population to date, there is a discrepancy between the percentages of $B R C A 1$ and $B R C A 2$ mutation carriers, and there is more likelihood of detecting gene mutations which have not been reported (Novel) or are variant of uncertain significance (VUS) (4). It is likely that as genetic testing gets more popularized, the VUS and novel mutation rates will decrease as we get to better understand our cohorts. The characterization of VUS pathogenicity can be based on different algorithms in different laboratories and centers and streamlining this particularly in Asia where there is still a comparative higher rate of detection of such VUS will aid the implementation of appropriate management of these families (21). Apart from $B R C A$ mutations, the inevitable use of gene panels to personalize care for individuals based on genetics would create a forward management options for families at risk. However, the validation and research work on these panels are warranted and is important based on the experience on $B R C A$ testing for use of the panels in ethnicities such as Asians, which to date are still relatively unknown in their world of genetic testing for alternative genes other than $B R C A$ genes.

Apart from the biology of genetic testing, practical issues such as genetic discrimination and cost of testing is still 
an imminent issue which would have to be confronted in Asia. Unlike the West, genetic testing is not covered by insurance nor government funds in Asia in general and most centers have obtained its resources for testing through research grants and philanthropy as an academic research project (19). Moreover there is no Genetic Information Non-discrimination act similar to that of United States (22) in most countries in Asia, hence unless this is addressed formally, there may be deterrent for genetic testing.

As genetic testing is gradually becoming an integral part of cancer care and to some, even considered as standard of care, for countries such as Asia where genetic testing and management for hereditary cancers are still comparatively at its infancy compared to the West, more attention should be given to performing research in this area and also implementation of a good supporting system in order to benefit more cancer patients and their families.

\section{Acknowledgements}

The author would also like to acknowledge the collaborative work of Members of the ABRCA Consortium and Team members of Division of Breast Surgery, The University of Hong Kong: in particular VY Shin, JW Chen, JC Ho and Department of Molecular Pathology, Hong Kong Sanatorium and Hospital, Hong Kong.

Funding: The author would like to acknowledge the funding support for work in Hong Kong by Hong Kong Hereditary Breast Cancer Family Registry, Hong Kong Sanatorium and Hospital, Dr. Ellen Li Charitable Foundation, the Kerry Group Kuok Foundation Limited and Health and Medical Research Fund, Hong Kong (1123176) and National Institute of Health 1R03CA130065.

\section{Footnote}

Conflicts of Interest: The author has no conflicts of interest to declare.

\section{References}

1. Hurley R. Angelina Jolie's double mastectomy and the question of who owns our genes. BMJ 2013;346:\{3340.

2. Miki Y, Swensen J, Shattuck-Eidens D, et al. A strong candidate for the breast and ovarian cancer susceptibility gene BRCA1. Science 1994;266:66-71.

3. Antoniou A, Pharoah PD, Narod S, et al. Average risks of breast and ovarian cancer associated with BRCA1 or BRCA2 mutations detected in case Series unselected for family history: a combined analysis of 22 studies. Am J Hum Genet 2003;72:1117-30.

4. Kwong A, Shin VY, Ho JC, et al. Comprehensive spectrum of BRCA1 and BRCA2 deleterious mutations in breast cancer in Asian countries. J Med Genet 2016;53:15-23.

5. Kwong A, Chen J, Shin VY, et al. The importance of analysis of long-range rearrangement of BRCA1 and BRCA2 in genetic diagnosis of familial breast cancer. Cancer Genet 2015;208:448-54.

6. Ou M, Ma R, Cheung J, et al. database.bio: a web application for interpreting human variations.

Bioinformatics 2015;31:4035-7.

7. Kwong A, Shin VY, Au CH, et al. Detection of Germline Mutation in Hereditary Breast and/or Ovarian Cancers by Next-Generation Sequencing on a Four-Gene Panel. J Mol Diagn 2016. [Epub ahead of print].

8. Kwong A, Shin VY, Cheuk IW, et al. Germline RECQL mutations in high risk Chinese breast cancer patients. Breast Cancer Res Treat 2016;157:211-5.

9. LaDuca H, Stuenkel AJ, Dolinsky JS, et al. Utilization of multigene panels in hereditary cancer predisposition testing: analysis of more than 2,000 patients. Genet Med 2014;16:830-7.

10. Tung N, Battelli C, Allen B, et al. Frequency of mutations in individuals with breast cancer referred for BRCA1 and BRCA2 testing using next-generation sequencing with a 25-gene panel. Cancer 2015;121:25-33.

11. Antoniou AC, Durocher F, Smith P, et al. BRCA1 and BRCA2 mutation predictions using the BOADICEA and BRCAPRO models and penetrance estimation in high-risk French-Canadian families. Breast Cancer Res 2006;8:R3.

12. Berry DA, Iversen ES Jr, Gudbjartsson DF, et al. BRCAPRO validation, sensitivity of genetic testing of BRCA1/BRCA2, and prevalence of other breast cancer susceptibility genes. J Clin Oncol 2002;20:2701-12.

13. Parmigiani G, Berry D, Aguilar O. Determining carrier probabilities for breast cancer-susceptibility genes BRCA1 and BRCA2. Am J Hum Genet 1998;62:145-58.

14. Frank TS, Deffenbaugh AM, Reid JE, et al. Clinical characteristics of individuals with germline mutations in BRCA1 and BRCA2: analysis of 10,000 individuals. J Clin Oncol 2002;20:1480-90.

15. Couch FJ, DeShano ML, Blackwood MA, et al. BRCA1 mutations in women attending clinics that evaluate the risk of breast cancer. N Engl J Med 1997;336:1409-15.

16. Shattuck-Eidens D, Oliphant A, McClure M, et al. BRCA1 
sequence analysis in women at high risk for susceptibility mutations. Risk factor analysis and implications for genetic testing. JAMA 1997;278:1242-50.

17. Kang E, Park SK, Lee JW, et al. KOHBRA BRCA risk calculator (KOHCal): a model for predicting BRCA1 and BRCA2 mutations in Korean breast cancer patients. J Hum Genet 2016;61:365-71.

18. Warner E, Hill K, Causer P, et al. Prospective study of breast cancer incidence in women with a BRCA1 or BRCA2 mutation under surveillance with and without magnetic resonance imaging. J Clin Oncol 2011;29:1664-9.

19. Nakamura S, Kwong A, Kim SW, et al. Current Status of the Management of Hereditary Breast and Ovarian Cancer in Asia: First Report by the Asian BRCA Consortium.

Cite this article as: Kwong A. Genetic testing for hereditary breast cancer in Asia-moving forward. Chin Clin Oncol 2016;5(3):47. doi: 10.21037/cco.2016.05.11
Public Health Genomics 2016;19:53-60.

20. Moyer VA; U.S. Preventive Services Task Force. Risk assessment, genetic counseling, and genetic testing for BRCA-related cancer in women: U.S. Preventive Services Task Force recommendation statement. Ann Intern Med 2014;160:271-81.

21. Maxwell KN, Hart SN, Vijai J, et al. Evaluation of ACMG-Guideline-Based Variant Classification of Cancer Susceptibility and Non-Cancer-Associated Genes in Families Affected by Breast Cancer. Am J Hum Genet 2016;98:801-17.

22. The Genetic Information Nondiscrimination Act of 2008. U.S. Equal Employment Opportunity Commission 2008:110-233. Available online: https://www.eeoc.gov/ laws/statutes/gina.cfm 